\title{
Inner Disk Structure of Dwarf Novae in the Light of X-Ray Observations
}

\author{
Ş. Balman ${ }^{1}$ \\ ${ }^{1}$ Middle East Technical University, Department of Physics, Inonu Bulvarı, Ankara, 06531, Turkey \\ Corresponding author: solen@astra.physics.metu.edu.tr
}

\begin{abstract}
Diversity of the X-ray observations of dwarf nova are still not fully understood. I review the X-ray spectral characteristics of dwarf novae during the quiescence in general explained by cooling flow models and the outburst spectra that show hard X-ray emission dominantly with few sources that reveal soft X-ray/EUV blackbody emission. The nature of aperiodic time variability of brightness of dwarf novae shows band limited noise, which can be adequately described in the framework of the model of propagating fluctuations. The frequency of the break $(1-6 \mathrm{mHz})$ indicates inner disk truncation of the optically thick disk with a range of radii (3.0-10.0) $\times 10^{9} \mathrm{~cm}$. The RXTE and optical (RTT150) data of SS Cyg in outburst and quiescence reveal that the inner disk radius moves towards the white dwarf and receeds as the outburst declines to quiescence. A preliminary analysis of SU UMa indicates a similar behaviour. In addition, I find that the outburst spectra of WZ Sge shows two component spectrum of only hard X-ray emission, one of which may be fitted with a power law suggesting thermal Comptonization occuring in the system. Cross-correlations between the simultaneous UV and X-ray light curves (XMM-Newton) of five DNe in quiescence show time lags in the X-rays of 96-181 sec consistent with travel time of matter from a truncated inner disk to the white dwarf surface. All this suggests that dwarf novae and other plausible nonmagnetic systems have truncated accretion disks indicating that the disks may be partially evaporated and the accretion may occur through hot (coronal) flows in the disk.
\end{abstract}

Keywords: cataclysmic variables - white dwarfs - accretion, accretion dics - stars: binaries - X-rays: stars - radiation mechanisms: thermal - stars: dwarf novae.

\section{Introduction}

Dwarf novae (DNe) are a class of cataclysmic variables $(\mathrm{CVs})$ which are interacting compact binaries in which a white dwarf (WD, the primary star) accretes matter and angular momentum from a main (or post-main) sequence star (the secondary) filling its Roche-lobe. The matter is transferred by means of an accretion disk that is assumed to reach all the way to the WD surface. Ongoing accretion at a low rate (quiescence) is interrupted every few weeks to months or sometimes with longer durations by intense accretion (outburst) of days to weeks where $\dot{M}$ increases (see Warner 1995 for a review).

The material in the inner disk of nonmagnetic CVs initially moving with Keplerian velocity dissipates its kinetic energy in order to accrete onto the slowly rotating WD creating a boundary layer (BL) (see Mauche 1997, Kuulkers et al. 2006 for an overview). Standard accretion disk theory predicts half of the accretion luminosity to originate from the disk in the optical and ultraviolet (UV) wavelengths and the other half to emerge from the boundary layer as X-ray and/or extreme UV $(\mathrm{EUV}) /$ soft X-ray emission which may be summerized as $\mathrm{L}_{B L} \sim \mathrm{L}_{d i s k}=\mathrm{GM}_{W D} \dot{M}_{a c c} / 2 \mathrm{R}_{W D}=\mathrm{L}_{a c c} / 2$ (Lynden-
Bell \& Pringle 1974, Godon et al. 1995). During low-mass accretion rates, $\dot{M}_{a c c}<10^{-(9-9.5)} \mathrm{M}_{\odot}$, the boundary layer is optically thin (Narayan \& Popham 1993, Popham 1999) emitting mostly in the hard Xrays $\left(\mathrm{kT} \sim 10^{(7.5-8.5)} \mathrm{K}\right)$. For higher accretion rates , $\dot{M}_{a c c} \geq 10^{-(9-9.5)} \mathrm{M}_{\odot}$, the boundary layer is expected to be opticallly thick (Popham \& Narayan 1995) emitting in the soft X-rays or EUV (kT 10 $\left.0^{(5-5.6)} \mathrm{K}\right)$. The transition between an optically thin and an optically thick boundary layer, also depends on the mass of the white dwarf (also rotation) and on the alpha viscosity parameter.

\section{X-Ray Observations of Dwarf Novae in Quiescence and Outburst}

The quiescent X-ray spectra are mainly characterised with a multi-temperature isobaric cooling flow model of plasma emission at $\mathrm{T}_{\max }=6-55 \mathrm{keV}$ with accretion rates of $10^{-12}-10^{-10} \mathrm{M}_{\odot} \mathrm{yr}^{-1}$. The X-ray line spectroscopy indicates narrow emission lines (brightest OVIII $\mathrm{K} \alpha$ ) and near solar abundances, with a $6.4 \mathrm{keV}$ line expected to be due to reflection from the surface of the WD. The detected Doppler broadening in lines during quies- 
Inner Disk Structure of Dwarf Novae in the Light of X-Ray Observations

cence is $<750 \mathrm{~km} \mathrm{~s}^{-1}$ at sub-Keplerian velocities in the boundary layer with electron densities $>10^{12} \mathrm{~cm}^{-3}$ (see Baskill et al. 2005, Kuulkers et al. 2006, Rana et al. 2006, Pandel et al. 2005, Balman et al. 2011, Balman 2012). The total X-ray luminosity during quiescence is $10^{29}-10^{32} \mathrm{erg} \mathrm{s}^{-1}$. Lack of BL emission in the $\mathrm{X}$ rays have been suggested due to low $\mathrm{L}_{x} / \mathrm{L}_{\text {disk }}$ ratio (see Kuulkers et al. 2006). It has been suggested for DN in quiescence that if the WD emission is removed and some disk truncation is allowed, this ratio is $\sim 1$ (Pandel et al. 2005).

DN outbursts are brightenings of the accretion disks as a result of thermal-viscous instabilities summerized in the DIM model (Disc Instability Model; Lasota 2001,2004). During the outburst stage, DN X-ray spectra differ from the quiescence since the accretion rates are higher (about two orders of magnitude), the BL is expected to be optically thick emitting EUV/soft Xrays (Lasota 2001, see the X-ray review in Kuulkers et al. 2006). On the other hand, soft X-ray/EUV temperatures in a range $5-25 \mathrm{eV}$ are detected from only about five systems (e.g., Mauche et al. 1995, Mauche \& Raymond 2000, Long et al. 1996, Mauche 2004, Byckling et al. 2009). As a second and more dominantly detected emission component, DN show hard X-ray emission during the outburst stage however, at a lower flux level and X-ray temperature compared with the quiescence (e.g., WW Cet \& SU UMa: Collins \& Wheatley 2010, Fertig et al. 2011, SS Cyg: Wheatley et al. 2003, MacGowen et al. 2004, Ishida et al. 2009). On the other hand, some DN show increased level of X-ray emission (GW Lib \& U Gem: Byckling et al. 2009, Guver et al. 2006). The total X-ray luminosity during outburst is $10^{30}-10^{34}$ $\mathrm{erg} \mathrm{s}^{-1}$. The grating spectroscopy of the outburst data indicate large widths for lines with velocities in excess of $1000 \mathrm{~km} \mathrm{~s}^{-1}$ mostly of $\mathrm{H}$ and He-like emission lines (C,N,O,Ne, Mg, Si, Fe, ect.) (Mauche 2004, Rana et al. 2006, Okada et al. 2008). A characteristic of some DN outburst light curves are the UV and X-ray delays in rise to outburst (w.r.t. optical) indicating possible disk truncation ((Meyer \& Meyer-Hofmeister 1994 and references therein). These delays are a matter of several hours (upto a day) that need dedicated simultaneous multi-wavelength observations. During outburst no eclipses are detected in the eclipsing systems (particularly of soft X-ray emission) or no distinct orbital variations are seen. (e.g., Pratt et al. 1999, Byckling et al. 2009). Note that both in SS Cyg (Mc Gowan et al. 2004) and in SU UMa (Collins \& Wheatley 2010) the X-ray flux in between outbursts have been found to decrease as opposed to expectations of the DIM model.

WZ Sge is a short period SU UMa type dwarf nova with long interoutburst interval of 20-30 yrs. The system is believed to show no normal outbursts but only superoutbursts. The most recent outburst in July-
August 2001 (previous outbursts in 1978, 1946 and 1913) was observed from ground and space over several different wavelength bands including the X-ray wavelengths and the EUV using the Chandra Observatory (see Wheatley \& Mauche 2005, Kuulkers et al. 2002). There has been three LETG (Low Energy Transmission Grating) and three ACIS-S (Advanced Camera Imaging Specreometer) observations obtained in the continuousclocking $(\mathrm{CC})$ mode. I report a preliminary analysis on the spectral fitting of the ACIS-S CC-mode data.

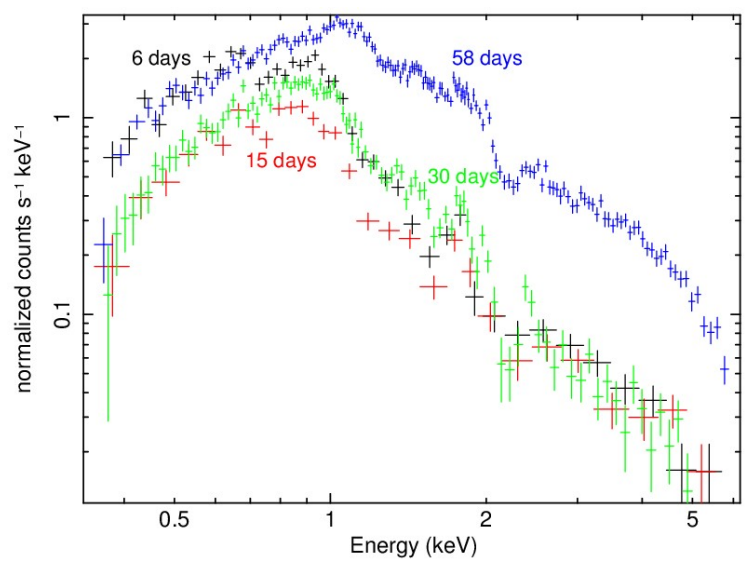

Figure 1: Chandra X-ray outburst spectra of WZ Sge in 0.3-10.0 keV range obtained with ACIS-S (CCmode). The observation days after the outburst are labeled on each spectrum.

The spectra and response files were prepared in a standard way (task SPECEXTRACT is used) by choosing the source and the background photons using a suitable extraction box (10 arcsec in size) on the data strip using CIAO 4.4 and the CALDB 4.4.8 (see http://cxc.harvard.edu/ciao/). For further analysis, HEASOFT version 6.13 is utilized. The spectra were fitted with the multi-temperature plasma emission models (e.g., CEVMKL in XSPEC, see http://heasarc.nasa.gov/xanadu/xspec/) according to the expections from earlier work of DN analysis in quiescence and outburst. CEVMKL model is a multitemperature plasma emission model (built from mekal code, Mewe et al. 1986) where Emission measures follow a power-law in temperature $\left(\mathrm{dEM}=(\mathrm{T} / \mathrm{Tmax})^{\alpha-1}\right.$ $\mathrm{dT} /$ Tmax). The reduced $\chi^{2}$ of the fits were above a value of 2 with either a single MEKAL model or CEVMEKL alone (the $\alpha$ parameter of the power law distribution of temperatures is set free) for days 6,15 , 30 after outburst. I added a second component of a power law which reduced the $\chi^{2}$ values to desirable levels. In general, the plasma has variable abundance of $\mathrm{N}, \mathrm{O}, \mathrm{Ne}, \mathrm{Fe}$, and $\mathrm{S}$. The spectral parameters for day 6 after the outburst were $\mathrm{kT}_{\max }=0.7-1.3 \mathrm{keV}$ with a photon index of $\Gamma=0.8-1.4$. The $\mathrm{X}$-ray flux of the ther- 
mal CEVMKL component was $1.1 \times 10^{-11} \mathrm{erg} \mathrm{s}^{-1} \mathrm{~cm}^{-2}$ and $\mathrm{L}_{\text {cevmkl }}$ is $2.7 \times 10^{30} \mathrm{erg} \mathrm{s}^{-1}$ where the power law component has $5.8 \times 10^{-12}$ erg s$^{-1} \mathrm{~cm}^{-2}$ and $\mathrm{L}_{\text {power }}$ is $1.3 \times 10^{30} \mathrm{erg} \mathrm{s}^{-1}$ (43.5 pc is assumed, Harrison et al. 2004). The parameters for day 15 were $\mathrm{kT}_{\max }=0.5-1.5$ $\mathrm{keV}$ and $\Gamma=0.2-1.1$. Between these two dates the $\mathrm{X}$-ray flux of the CEVMKL component decreased by 2 and the power law component inceased by about 1.4. For day $30 \mathrm{kT}_{\max }=1.2-3.0 \mathrm{keV}$ and $\Gamma=1.5-2.0$. Therefore, the $\mathrm{X}$-ray emitting region gets hotter and the photon index decrease. The X-ray fluxes recover to day 6 . The final observation on day 58 which is almost quiescence after the outburst shows $\mathrm{kT}_{\max }=26-46 \mathrm{keV}$ and no power law emission is detected. The $\mathrm{X}$-ray flux is the highest $4.4 \times 10^{-11} \mathrm{erg} \mathrm{s}^{-1} \mathrm{~cm}^{-2}$ and $\mathrm{L}_{x}$ is $1.1 \times 10^{31} \mathrm{erg} \mathrm{s}^{-1}$ (at $43.5 \mathrm{pc}$ ). All ranges correspond to $90 \%$ confidence level errors. The neutral hydrogen absorption has stayed at the interstellar level in all fits within errors. No optically thick blackbody emission is detected and only $\mathrm{X}$-ray suppression of the quiescent emission is observed just like some other DN. The X-ray temperatures on days 6-30 are in good agreement with the LETG results as well. A model of three additive MEKALs yield acceptable fits on days 6-30, however allowing for very high X-ray tempartures. A partial covering absorber model improves the CEVMKL fits, however the high intrinsic absorption when modeled yields inconsistent count rates for the LETG observations.

\section{Inner Disk Structure Using Eclipse Mapping Techniques}

Flickering is a fast intrinsic brightness scintilation occurring on time scales from seconds to minutes (e.g., amplitudes of 0.01 - $1 \mathrm{mag}$ in the optical). It is observed in all accreting sources. Eclipse mapping methods have been used to reproduce the spatial distribution of flickering in CVs in the optical and UV wavelengths. Some eclipse mapping studies of flickering in quiescent dwarf novae indicate that mass accretion rate diminishes by a factor of 10-100 and sometimes by 1000 in the inner regions of the accretion disks as revealed by the brightness temperature calculations which do not find the expected $\mathrm{R}^{-3 / 4}$ radial dependence of brightness temperature in standard steady-state constant mass accretion rate disks (see e.g., Z Cha and OY Car: Wood 1990, V2051 OPh: Baptista \& Bortoletto 2004, V4140 Sgr: Borges \& Baptista 2005). Biro (2000) finds that this flattening in the brightness temperature profiles may be lifted by introducing disk truncation in the quiescent state (e.g., $\mathrm{r} \sim 0.15 \mathrm{R}_{L 1} \sim 4 \times 10^{9} \mathrm{~cm}$; DW UMa, a nova-like). A comprehensive UV modeling of accretion disks at high accretion rates in $33 \mathrm{CV}$ including many nova-likes and old novae (Puebla et al. 2007) indicate an extra component from an extended optically thin region (e.g., wind, corona/chromosphere) evident from the strong emission lines and the P Cygni profiles. This study also indicates that the mass accretion rate may be decreasing 1-3 orders of magnitude in the inner disk region (divergence starts $\mathrm{r} \leq$ afew $\times 10^{10} \mathrm{~cm}$ towards the WD).

\section{Inner Disk Structure of Dwarf Novae}

The truncation of the optically thick accretion disk in DNe in quiescence was invoked as a possible explanation for the time lags between the optical and UV fluxes in the rise phase of the outbursts (Meyer \& MeyerHofmeister 1994), and for some implications of the DIM (see Lasota 2001, 2004) or due to the unusual shape of the optical spectra or light curves of DNe (Linell et al. 2005, Kuulkers et al. 2011). Theoretical support for such a two-phase flow was given by a model of the disk evaporation of (Meyer \& Meyer-Hofmeister 1994). This model was later elaborated to show that the disk evaporation (coronal "syphon" flow) may create optically thick-optically thin transition regions at various distances from the WD ( Liu et al. 1997, Mineshige et al. 1998).

\subsection{Propagating fluctuations model}

Another diagnostic tool proposed to study the inner disk structure in accreting objects is the aperiodic variability of brightness of sources in the X-rays. While the long time-scale variability might be created in the outer parts of the accretion disk (Warner \& Nather 1971), the relatively fast time variability (at $f>$ few $\mathrm{mHz}$ ) originates in the inner parts of the accretion flow (Bruch 2000; Baptista \& Borteletto 2004). Properties of this noise is similar to that of the X-ray binaries with neutron stars and black holes. Now, the widely accepted model of origin for this aperiodic flicker noise is a model of propagating fluctuations (Lyubarskii 1997, Churazov et al. 2001, Uttley \& McHardy 2001, Revnivtsev et al. 2009,2010, Uttlet et al. 2011, Scaringi et al. 2012). The modulations of the light are created by variations in the instantaneous value of the mass accretion rate in the region of the energy release. These variations in the mass accretion rate, in turn, are inserted into the flow at all Keplerian radii of the accretion disk due to the stochastic nature of its viscosity and then transferred toward the compact object. Thus, variations are on dynamical timescales. This model predicts that the truncated accretion disk should lack some part of its variability at high Fourier frequencies, i.e. on the time scales shorter than a typical time scale of variability. 


\subsection{Broad-band noise of dwarf novae}

A recent work by Balman \& Revnivtsev (2012) have used the broad-band noise characteristic of selected DN in quiescence (only one in outburst: SS Cyg) and studied the inner disk structure and disk truncation via propagating fluctuations model. The power spectral densities (PDS) expressed were calculated in terms of the fractional rms amplitude squared following from (Miyamoto et al. 1991). The light curves were divided into segments using 1-5 sec binning in time and several PDS were averaged to create a final PDS of the sources. The white noise levels were subtracted hence leaving us with the rms fractional variability of the time series in units of $(\mathrm{rms} / \mathrm{mean})^{2} / \mathrm{Hz}$. Next, the rms fractional variability per hertz was multiplied with the frequencies to yield $\nu P_{\nu}$ versus $\nu$. For the model fitting a simple analytical model is used

$$
P(\nu) \propto \nu^{-1}\left(1+\left(\frac{\nu}{\nu_{0}}\right)^{4}\right)^{-1 / 4}
$$

, which was proposed to describe the power spectra of sources with truncated accretion disks (see Revnivtsev et al. 2010,2011). The broad-band noise structure of the Keplerian disks often show $\propto f^{-1 \ldots-1.3}$ dependence on frequency (Churazov et al. 2001, Gilfanov et al. 2005), and this noise will show a break if the optically thick disk truncates as the Keplerian motion subsides.

Balman \& Revnivtsev (2012) show that for five DN systems, SS Cyg, VW Hyi, RU Peg, WW Cet and T leo, the UV and X-ray power spectra show breaks in the variability with break frequencies in a range 1-6 $\mathrm{mHz}$, indicating inner disk truncation in these systems. The truncation radii for DN are calculated in a range $\sim(3-$ 10) $\times 10^{9} \mathrm{~cm}$ including errors (see Table 2 in Balman \& Revnivtsev 2012).

The same authors used the archival RXTE data of SS Cyg in quiescence and outburst listed in Table 1 of their paper to derive the broad-band noise of the source in different states (i.e., accretion rates). For the outburst phases, authors investigated times during the X-ray suppression (e.g., the X-ray dips; optical peak phases of the outburst) and the X-ray peak. This, in turn is expected to indicate the motion of the flow in the inner regions of the disk and the geometry of the inner disk. Authors show that the disk moves towards the white dwarf during the optical peak to $\sim 1 \times 10^{9} \mathrm{~cm}$ and receeds as the outburst declines to quiescence to $5-6 \times 10^{9} \mathrm{~cm}$. This is shown for a CV, observationally, for the first time in the X-rays (see Figure 2). This is also supported with the optical data analysis of SS Cyg in quiescence and outburst (Revnivtsev et al. 2012)

\subsection{X-ray and UV light curve cross-correlations}

Balman \& Revnivtsev (2012) calculated the crosscorrelation between the two simultaneous light curves (X-ray and UV), using the archival XMM-Newton EPIC pn and OM data utilizing HEAsOFT task CROssCOR. To obtain the CCFs (cross-correlation functions), datasets were divided into several pieces using 1-5 sec binning in the light curves and fitted the resulting CCFs with double Lorentzians since it was necessary for adequate fitting (see the paper for details). The CCFs for all the DNe show clear asymmetry indicating that some part of the UV flux is leading the X-ray flux. In addition, they detect a strong peak near zero time lag for RU Peg, WW Cet and T Leo, suggesting a significant zero-lag correlation between the X-rays and the UV light curves.

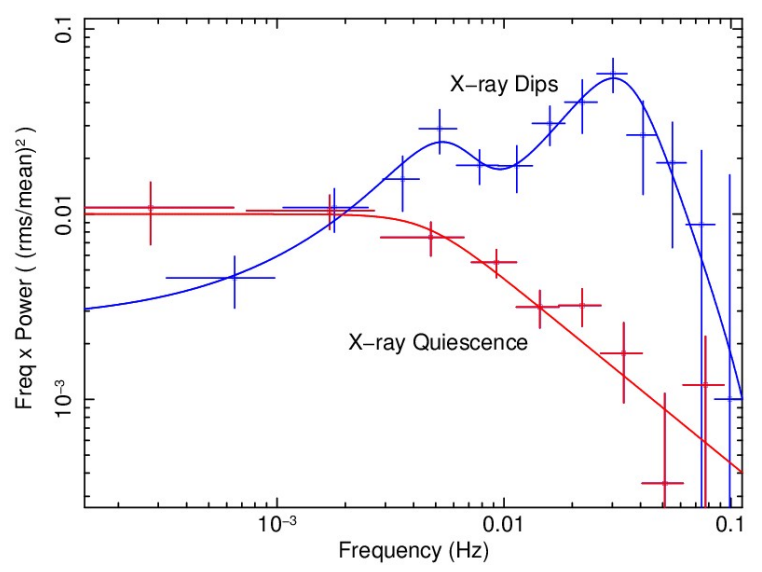

Figure 2: The RXTE PDS of SS Cyg in quiescence and during the optical peak of outburst (top). The solid lines show the fit with the propagating fluctuations model along with two Lorentzians.

The same authors also calculated the crosscorrelation between the simultaneous UV and X-ray light curves by subtracting the zero time lag components in the five DNe PDS, yielding more correct time lags consistent with delays in the X-rays of 96-181 sec (see the paper on details of the modeling). The lags occur such that the UV variations lead X-ray variations which shows that as the accreting material travels onto the WD, the variations are carried from the UV into the $\mathrm{X}$-ray emitting region. Therefore, the long time lags of the order of minutes can be explained by the travel time of matter (viscous flow) from a truncated inner disk to the white dwarf surface. Zero time lags ( light travel time) indicate irradiation effects in these systems since we do not have resolution better than $1 \mathrm{sec}$. 

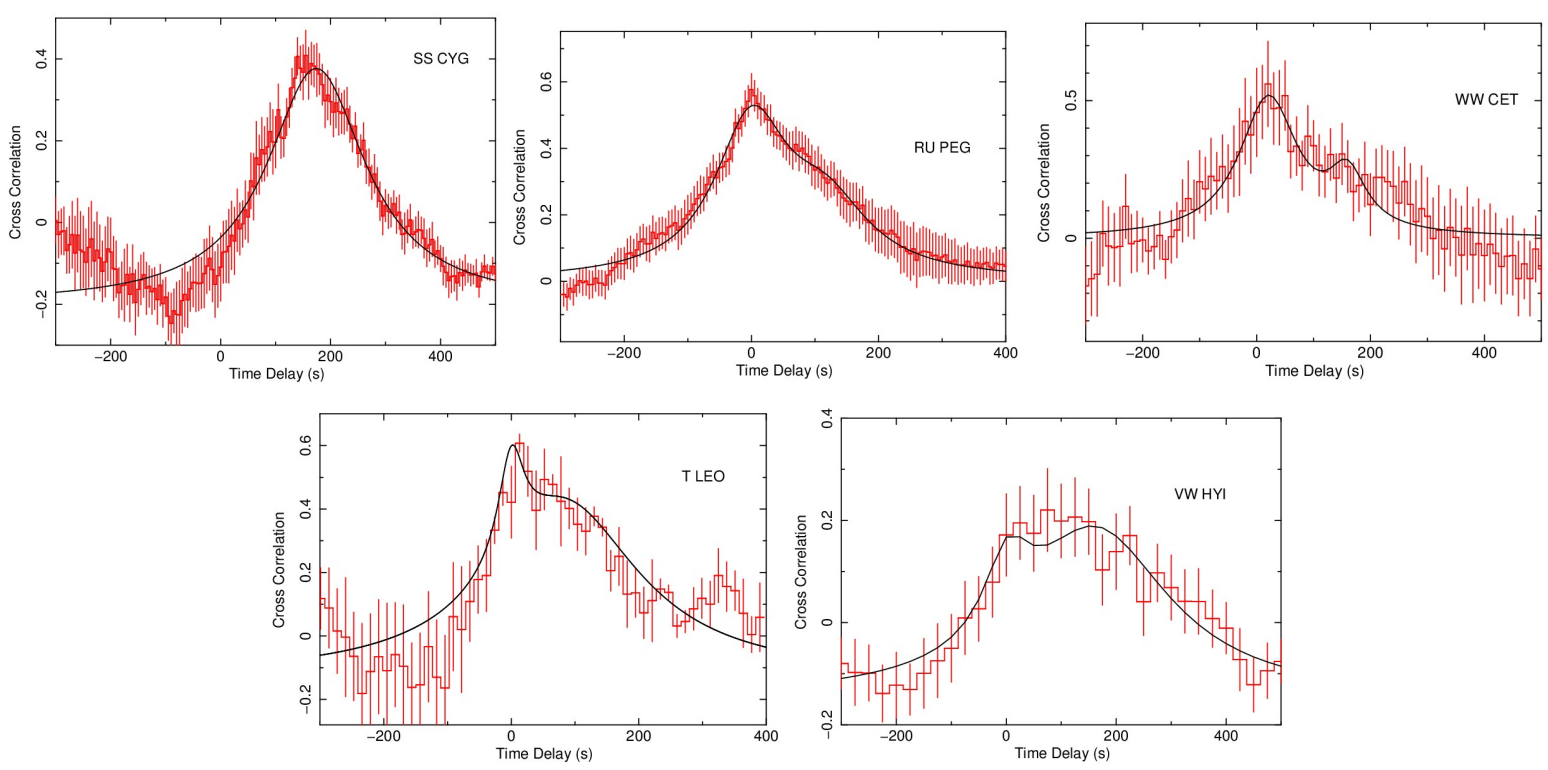

Figure 3: See Figure 10 in Balman \& Revnivtsev (2012). The cross-correlation of the EPIC pn (X-ray) and OM (UV) light curves with 1 sec time resolution. The two-component Lorenzian fits are shown as solid black lines (except for SS Cyg).
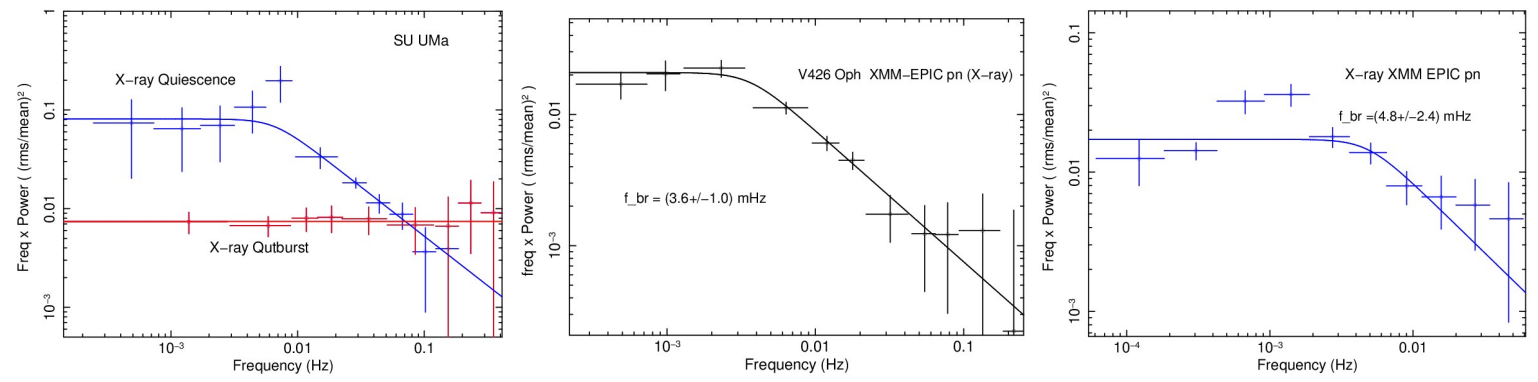

Figure 4: The RXTE PDS of SU UMa in quiescence and in optical outburst is displayed (top panel). $X M M$ Newton EPIC pn PDS of V426 Oph and HT Cas in quiescence (middle and bottom panel). The solid lines are the fits with the propagating fluctuations model.

An analysis on the RXTE data of SU UMa in quiescence and outburst following six consecutive outburts reveal a similar broad-band noise structure to SS Cyg in quiescence showing a break frequency $\sim 5.5-7.5 \mathrm{mHz}$ with a truncated optically thick disk $\sim 3.8 \times 10^{9} \mathrm{~cm}$. The preliminary analysis of the outburst data during the X-ray suppression episodes (optical peak of the outburst) indicates no disk truncation or a truncation around $0.1 \mathrm{~Hz}$ (see Figure 4).

Therefore, SU UMa indicates a similar behaviour of the disk during the outburst to SS Cyg where the inner disk moves in almost all the way to the WD during the optical peak and moves out in decline to quiescent location further out. The lower level of broadband noise during outburst stage of SU UMa may be due to the high radiation pressure support of an opti- cally thick disk flow during the peak of the outburst suppressing the variations. A similar PDS analysis of the XMM-Newton data of the DNe, V426 Oph and HT Cas in quiescence reveals break frequencies 4.6-2.6 $\mathrm{mHz}$ and $7.2-2.8 \mathrm{mHz}$, respectively. The approximate Keplerian radii where the optically thick disk truncates is $\sim 6.2 \times 10^{9} \mathrm{~cm}$ and $\sim 4.5 \times 10^{9} \mathrm{~cm}$, respectively (see Figure 4).

\section{Conclusions}

Studies of DNe broad-band noise characteristics in the X-rays (see also Balman \& Revnivtsev 2012) indicate that $\mathrm{DNe}$ have truncated accretion disks in quiescence detected in at least 8 systems in a range $\sim(3.0-10.0) \times 10^{9} \mathrm{~cm}$ including errors. The Magnetic CVs (MCVs) show rather smaller truncation radii (0.9- 
Inner Disk Structure of Dwarf Novae in the Light of X-Ray Observations

$2.0) \times 10^{9} \mathrm{~cm}$ (Revnivtsev et al. 2010, 2011). This can also explain the UV and X-ray delays in the outburst stage and the accretion may occur through hot (coronal) flows in the disk. Note that extended emission and winds are detected from DN in the outburst stage which may be an indication of the coronae/hot flows in these systems (e.g., Mauche 2004). Time delays detected in a range of $96-181 \mathrm{sec}$, are also consistent with matter propogation timescales onto the WD in a truncated nonmagnetic CV disk in quiescence. Balman \& Revnivtsev (2012) approximate an $\alpha$ of 0.1-0.3 for the inner regions of the DNe accretion disks in quiescence.

In addition, the outburst spectra of WZ Sge shows two component spectrum of only hard X-ray emission, one of which may be fitted with a power law suggesting thermal Comptonization of the optically thick disk photons or scattering from an existing wind during the outburst. The spectral evolution and disapearance of the power law component after outburst may support this issue and that the accretion disk goes back to its quiescent truncated structure and Comptonization stops.

A general picture of the accretion flow around a WD in quiescence thus might be somewhat similar to that of the black hole/neutron star accretors with an optically thick colder outer accretion disk and an optically thin hot flow in the inner regions where the truncation occurs (see Esin et al. 1997, Done et al. 2007). The appearance of a hot flow (e.g., ADAF-like) in the innermost regions of the accretion disk will differ from that of ordinary rotating Keplerian disk because it is no longer fully supported by rotation, but might have a significant radial velocity component with sub-Keplerian speeds.

It is important to monitor DNe in the X-rays to measure the variability in the light curves in time together with the variations of the possible disk truncation and formation of plausible coronal (optically thin) and/or ADAF-like flows/regions on the disk in quiescence and outburst.

\section{Acknowledgement}

SB thanks to M. Revnivtsev for his valuable collaboration on the timing analysis of DN.

\section{References}

[1] Balman, S., Godon, P., Sion, E.M., Ness, J.-U., Schlegel, E., Barrett, P.E., \& Szkody, P.: 2011, ApJ, 741, 84.

[2] Balman, S., \& Revnivtsev, M.: 2012, A\&A, 546, 112.

[3] Balman, S.: 2012, MmSAI, 83, 585
[4] Baptista, R., Bortoletto A.: 2004, AJ, 128, 411.

[5] Borges, B.W., Baptista, R.: 2005, A\&A, 437, 235.

[6] Baskill, D., Wheatley, P.J., \& Osborne, J.P.: 2005, MNRAS, 357, 626. doi:10.1111/j.1365-2966.2005.08677.x

[7] Biro, I.B.: 2000, A\&A, 364, 573.

[8] Bruch A., 2000: A\&A, 359, 998.

[9] Byckling, K., et al.: 2009 MNRAS, 399, 1576. doi:10.1111/j.1365-2966.2009.15378.x

[10] Churazov, E., Gilfanov, M., \& Revnivtsev, M.: 2001, MNRAS, 312, 759. doi:10.1046/j.1365-8711.2001.04056.x

[11] Collins, D., J., \& Wheatley, P. J.: 2010, MNRAS, 402, 1816

[12] Done, C., Gierliński, M., Kubota, A.: 2007, A\&ARv, 15, 1.

[13] Esin, A. A., McClintock, J. E., Narayan, R.: 1997, ApJ, 489, 865.

doi:10.1086/661949

[14] Fertig, D., Mukai, K., Nelson, T., \& Cannizzo, J.K.: 2011, PASP, 123, 1054.

[15] Gilfanov, M., \& Arefiev, V.: 2005, astro, arXiv:astro-ph/0501215 doi:10.1093/mnras/275.4.1093

[16] Godon, P., Regev, O., \& Shaviv, G.: 1995, MNRAS, 275, 1093. doi:10.1111/j.1365-2966.2006.10896.x

[17] Guver, T., Uluyaz,E., Ozkan, M. T., Gogus, E.: 2006, MNRAS, 372, 450.

[18] Harrison, T. E., Johnson, J.J., McArthur., B.E., Benedict, G.F., Szkody, P., Howell, S.B., Gelino, D.M.: 2004 AJ, 127, 460.

[19] Ishida, M., Okada, S., Hayashi, T., Nakamura, R., Terada, Y., Mukai, K., \& Hamaguchi, K.: 2009, PASJ, 61, 77.

[20] Kuulkers, E., Knigge, C., Steeghs, D., Wheatley, P. J., \& Long, K. S.: 2002, in The Physics of Cataclysmic Variables and Related Objects, B. T. Gnsicke, K. Beuermann, and K. Reinsch(ed.), ASP Conf. Ser. Vol. 261, San Francisco: Astronomical Society of the Pacific, p. 443.

[21] Kuulkers, E., Norton, A., Schwope, A., \& Warner, B.: 2006, in Compact stellar X-ray sources, W. Lewin \& M. van der Klis (ed.), Cambridge Astrophysics Series No. 39 (Cambridge, UK: Cambridge University Press), p. 421. 
[22] Kuulkers, E., Henden, A. A., Honeycutt, R. K., Skidmore, W., Waagen, E. O., \& Wynn G. A.: 2011, A\&A, 528, 152.

[23] Lasota, J. P.: 2001, NewAR, 45, 449

[24] Lasota, J. P.: 2004, RMxAC, 20, 124

[25] Linnell, A. P., Szkody, P., Gänsicke, B., Long, K. S., Sion, E. M., Hoard, D. W., \& Hubeny, I.: 2005, ApJ, 624, 923.

[26] Liu B. F., Meyer F., \& Meyer-Hofmeister E.: 1997, A\&A, 328, 247

[27] Long, K. S., Mauche, C., W., Raymond, J., C., Szkody, P., \& Mattei, J. A.: 1996, ApJ, 469, 841.

[28] Lynden-Bell, D. \& Pringle, J. E.: 1974, MNRAS, 168, 603. doi:10.1086/421438

[29] Lyubarskii, Y. E.: 1997, MNRAS, 292, 679. doi:10.1086/309489

[30] Mauche, C. W.: 2004, ApJ, 610, 422.

[31] Mauche, C. W., \& Raymond, J. C.: 2000, ApJ, 541,924 .

[32] Mauche, C. W.: 1997, in X-ray imaging and spectroscopy of hot plasmas, F. Makino \& K. Mitsuda (ed.), Proceedings of an International Symposium on X-ray Astronomy ASCA Third Anniversary, p. 529.

[33] Mauche, C. W., Raymond, J. C., \& Mattei, J. A.: 1995, ApJ, 446, 842. doi:10.1086/380758

[34] Mewe, R., Lemen, J.R., van den Oord, \& G.H.J.: 1986, A\&AS, 65, 511.

[35] McGowan, K.E., Priedhorsky, W.C., \& Trudolyubov, S. P.: 2004, ApJ, 601, 1100.

[36] Meyer, F., \& Meyer-Hofmeister, E.: 1994, 288, 175.

[37] Mineshige, S., Liu, B., Meyer, F., \& Meyer-Hofmeister, E.: 1998, PASJ, 50, L5. doi:10.1038/362820a0

[38] Miyamoto, S., Kimura, K., Kitamoto, S., Dotani, T., \& Ebisawa, K.: 1991, ApJ, 383, 784. doi:10.1086/587161

[39] Narayan, R., \& Popham, R.: 1993, Nature, 362, 820. doi:10.1086/429983

[40] Okada, S., Nakamura, R., \& Ishida, M.: 2008, ApJ, 680, 695.
[41] Pandel, D., Córdova, F.A., Mason, K.O., \& Priedhorsky, W.C.: 2005, ApJ, 626, 396.

[42] Pratt, G. W., Hassall, B.M., Naylor, T., Wood, J. H., \& Patterson, J.: 1999, MNRAS, 309, 847

[43] Popham, R., \& Narayan, R.: 1995, ApJ, 442, 337.

[44] Popham, R.: 1999, MNRAS, 308, 979.

[45] Puebla, R.E., Diaz, M.P., Hubney, I.: 2007, ApJ, 134, 1923.

[46] Rana, V. R., Singh, K. P., Schlegel, E. M., \& Barrett, P. E.: 2006, AdSpR, 38, 2847.

[47] Revnivtsev, M., Churazov, E., Postnov, K., \& Tsygankov, S.: 2009, A\&A, 507, 1211.

[48] Revnivtsev, M., et al.: 2010, A\&A, 513, 63.

[49] Revnivtsev, M., Potter, S., Kniazev, A., Burenin, R., Buckley, D.A.H., \& Churazov, E.: 2011, MNRAS, 411, 1317. doi:10.1111/j.1365-2966.2012.20512.x

[50] Revnivtsev, M. et al.: 2012, AstL, 38, 271. doi:10.1046/j.1365-8711.2001.04496.x

[51] Scaringi, S., Krdin,g E., Uttley, P., Knigge, C., Groot, P. J.,\& Still, M.: 2012, MNRAS, 421, 2854. doi:10.1111/j.1745-3933.2011.01056.x

[52] Uttley, P., \& McHardy, I. M.: 2001, MNRAS, 323, L26. doi:10.1017/CB09780511586491

[53] Uttley, P., Wilkinson T., Cassatella, P., Wilms J., Pottschmidt, K., Hanke, M., Böck, M.: 2011, MNRAS, 414, L60.

doi:10.1093/mnras/152.2.219

[54] Warner, B., 1995, Cataclysmic Variable Stars, Cambridge Univ. Press, Cambridge

[55] Warner, B., Nather, R.E., 1971, MNRAS, 152, 219. doi:10.1111/j.1365-2966.2003.07149.x

[56] Wheatley, P.J., \& Mauche, C.W.: 2005, in The Astrophysics of Cataclysmic Variables and Related Objects, J.-M. Hameury and J.-P. Lasota (ed.), Proc. Ser. ASP Conf. Vol.330, San Francisco: Astronomical Society of the Pacific, p. 257 doi:10.1086/317016

[57] Wheatley, P.J., Mukai, K., \& de Martino, D.: 2003, MNRAS, 346, 855.

[58] Wilms, J., Allen, A., \& McCray, R. 2000, ApJ, 542,914

[59] Wood, J. H.: 1990, MNRAS, 243, 219 